TITLE:

\title{
Mechanisms of Microearthquakes and Their Relation to Geological Structures
}

\section{$\operatorname{AUTHOR}(\mathrm{S}):$}

KISHIMOTO, Yoshimichi; NISHIDA, Ryohei

\section{CITATION:}

KISHIMOTO, Yoshimichi ...[et al]. Mechanisms of Microearthquakes and Their Relation to Geological Structures. Bulletin of the Disaster Prevention Research Institute 1973, 23(1): 125

ISSUE DATE:

1973-07

URL:

http://hdl.handle.net/2433/124831

RIGHT: 


\title{
Mechanisms of Microearthquakes and Their Relation to Geological Structures
}

\author{
By Yoshimichi KISHIMOTO and Ryohei NISHIDA
}

(Manuscript received July 17, 1973)

\begin{abstract}
For the purpose of examining relations between mechanism of microearthquakes and geological structure and also of investigating tectonic significance of microseismicity, 130 microearthquakes were analysed in an area surrounding the Yamasaki fault, which is a typical strikeslip fault in the northwestern Kinki District, Southwest Japan.

Mechanisms of microearthquakes were analysed by the method of fault plane solution for 69 earthquakes out of 130 , and by the smoothed radiation pattern using all earthquakes.

It is remarkable that along such a clear strike-slip fault as the Yamasaki fault almost all earthquakes have strike-slip type and their maximum pressure axes lie in a nearly $\mathrm{E} \cdot \mathrm{W}$ direction. This result regarding microearthquakes is coincident with thase for large and moderate earthquakes. Moreover, the strike of one nodal plane is nearly coincident with the fault strike, and it changes consistently when the fault strike changes its direction. On the other hand, in a microseismic belt along IZ-HM line (Fig. 1) where any clear strike-slip fault has not yet been detected, mechanisms of microearthquakes were very complicated, including strkie-slip, thrust and normal fault types.

These results suggest that the mechanism of the microearthquake, as well as its spacial distribution, is closely related to the geological structures.
\end{abstract}

\section{Introduction}

A fundamental pattern of spacial distribution of microearthquakes has been clarified rapidly in this decade in the northwestern part of Kinki, Southwest Japan. In 1965, the Tottori Microearthquake Observatory, Disaster Prevention Research Institute, Kyoto University completed the construction of its network and commenced routine observation of microseismicity in an area extending over Tottori, Okayama, Hyogo, Osaka and Kyoto Prefectures. In Fig. 1, the network is shown, with the microseismicity in this area, in which the network of the Abuyama Seismological Observatory is also denoted by the black triangle. Since the beginning of observation, a most remarkable observational fact was that epicenter distribution in this area showed a characteristic pattern, and many investigations have been published ${ }^{13,2), 3)}$ on this point.

Fig. 1 shows microseismicity from the latter half of 1964 to the middle of 1968. Also in Fig. 2, a recent microseismicity for about 3 years from 1969 to 1972, in which microseismicity only in nighttime from $8 \mathrm{p} . \mathrm{m}$. to $8 \mathrm{a} . \mathrm{m}$. is presented in order to ex- 


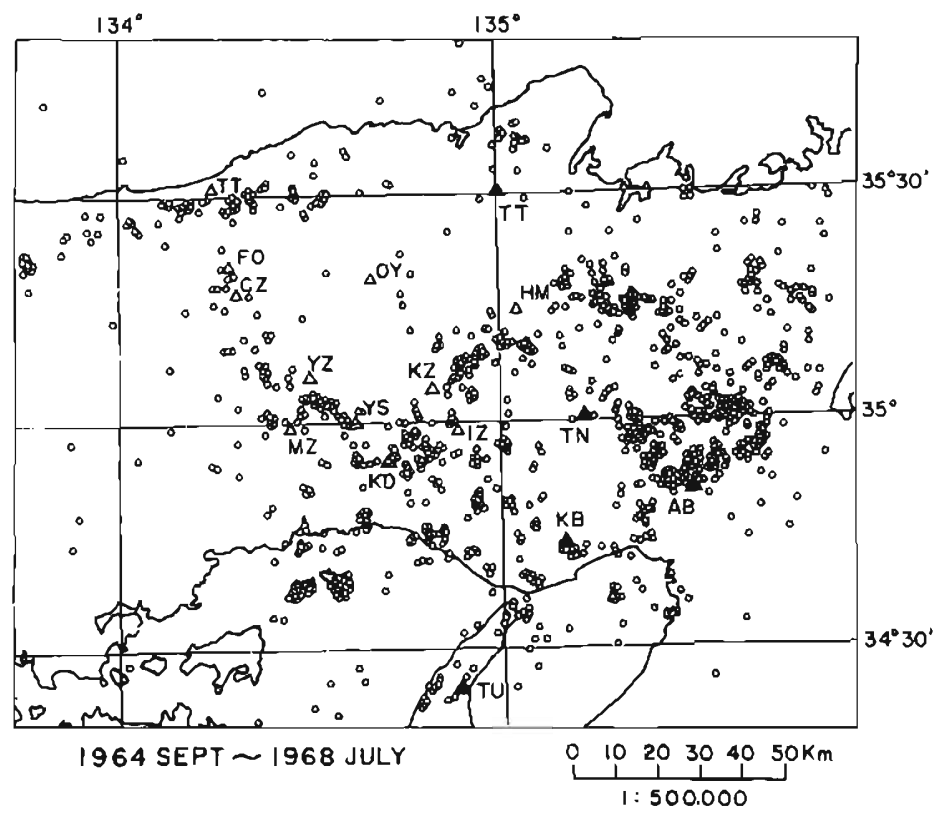

Fig. 1. Stationary activity of microearthquakes in the northwestern Kinki and the stations used in this analysis. White and black triangles denote the networks belonging to the Tottori Microearthquake Observatory and the Abuyama Seismological Observatory, respectively. Four stations, YZ, YS, KD, KZ, are temporary stations.

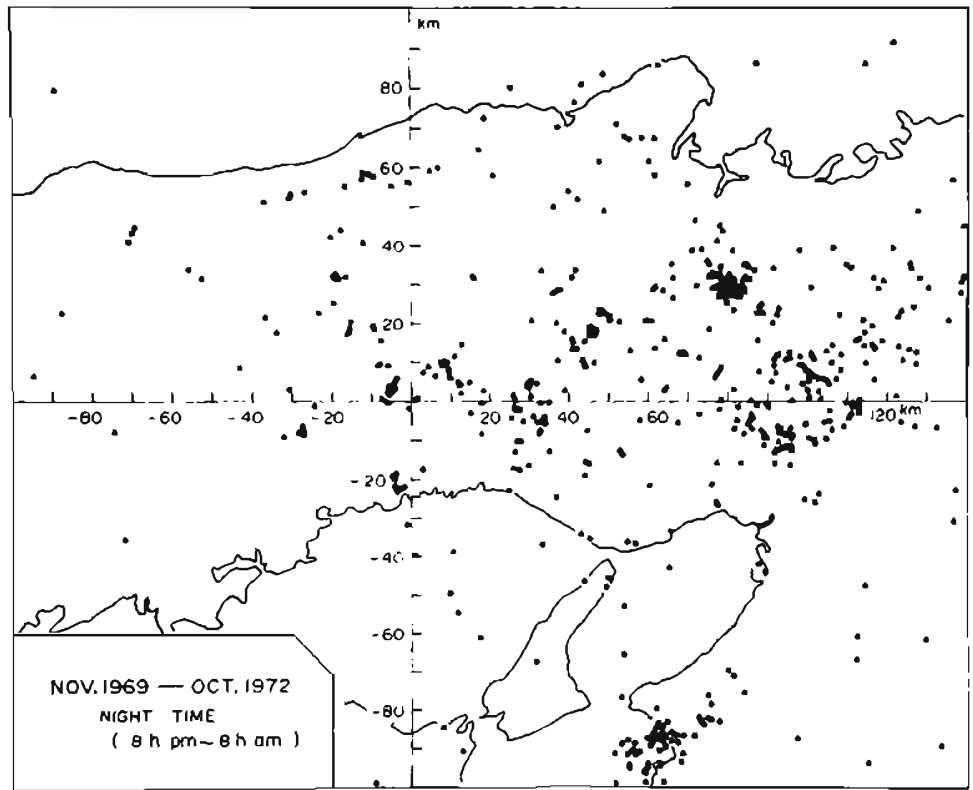

Fig. 2. Epicenter distribution of microearthquakes in nighttime in recent period. 


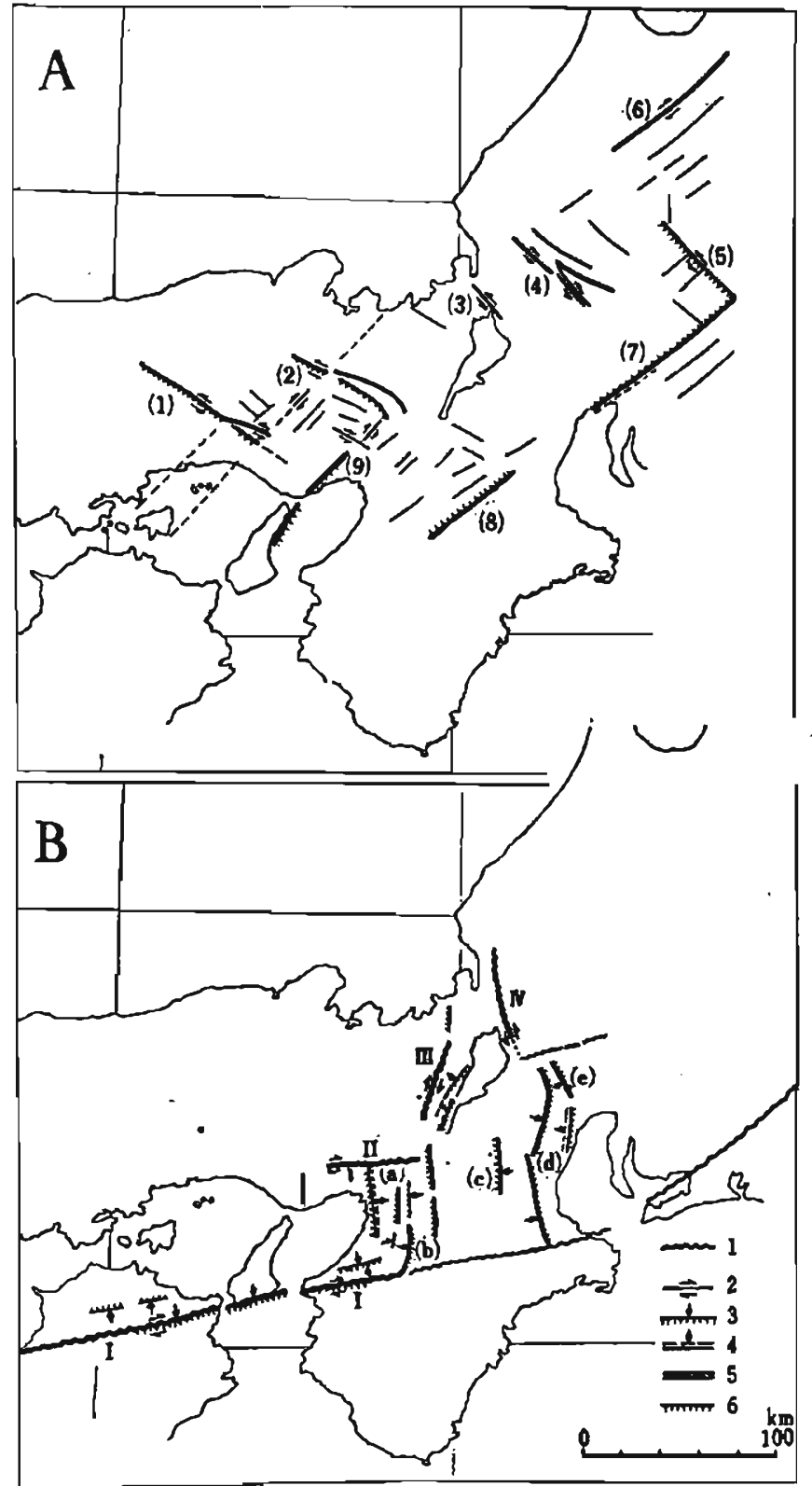

Fig. 3. Fault systems in Southwest Japan. A shows conjugate sets of strike-slip faults: (1); Yamasaki fault, (2); Mitoke fault, (6); Atotsugawa fault. B shows thrust faults of N-S trend, and tectonic lines (I to IV). (after Huzita et al.) 
clude the artificial explosions such as rock burst, dam construction and others. However, we can recognize no definite change of pattern of epicenter distribution between two figures, so that we may safely consider that these figures represent the stationary activity of microearthquakes in this area.

We shall here describe briefly about this characteristic pattern again, although it has been mentioned repeatedly in several papers, because this recognition is the starting point of our discussions. In this area, microearthquakes are generated in several belt-like zones, trends of which are nearly NW-SE or NE-SW. This pattern of distribution can be observed in Figs. 1 and 2. These belt-like zones intersect, forming rhombic aseismic blocks of various sizes.

On the other hand, investigations of geological structures in the northwestern Kinki in recent geological ages, including faults, foldings and terraces, have made rapid progress in these years. The progresses in both fields, seismology and neotectonics, have revealed a surprising correlation between microseismicity, which is naturally considered just a present phenomenon in the crust, and geological structures which have been built up over long geologic time.

First, we can recognize a clear coincidence between the belt-like zones of high seismicity in Figs. 1 and 2 and distribution of active faults shown in Fig. 3 . For instance, the Yamasaki and the Mitoke faults which are both typical strike-slip faults coincide well with two belt-like zones of high microseismicity. A great many earthquakes occur along west bank of the Yodo River, which makes conjugate direction with the Yamasaki fault. According to Huzita ${ }^{4)}$, the southeastern margin of this broad seismic belt corresponds to a boundary between two large geologic blocks, one being the Tamba Paleozoic Zone on the north side and the other "Kinki Triangle" composed of granitic rocks on the south side.

Crenerally speaking, in the Paleozoic zones, strike-slip faults such as the Yamasaki and Mitoke are prevailing, accompanying linear distribution of microearthquakes. This phenomenon is observable at other strike-slip faults, such as the Atotsugawa fault in the Chubu District.

Such close relations between microseismicity and geological structure may be one of the most important results so far obtained by investigations of microearthquakes. And this fact will have possibility to make clear mechanisms of earthquake generation and tectonic movement, and the relations between them.

In this article, we shall adopt the Yamasaki fault and its neighbouring areas as a model zone, and try in some detail to detect the relations between mechanisms of microearthquakes and geological structures, with an attempt mentioned just above.

\section{Mehanism of Microearthquakes}

Ichikawa55,6) investigated mechanisms of earthquakes of large and moderate magnitudes in Southwest Japan, using observational data by JMA. His result denotes that very shallow earthquakes (in the crust) in the Inner Zone have focal mecha- 
nisms of four-quadrant types with vertical null vector, and moreover that the direction of the maximum compression axis is E-W. In Fig. 4, distribution of the horizontal direction of the maximum pressure axis is shown. As mentioned earlier, observation of microearthquakes has shown rapid progress in these years, and many investigations of the mechanism of microearthquakes in the northwestern Kinki have been published 7),8, 9). They give an interesting result that the mechanisms of small and microearthquakes are the same as those of larger earthquakes treated by Ichikawa.

Recently, one of the authors ${ }^{10}$ ) investigated mechanisms of small and microearthquakes $(M \geqslant 2.5)$ in the northwestern part of Kinki. His analyses were carried out by two methods, namely one is the fault plane solutions of respective earthquakes and another is detection of mean tectonic stress in respective small regions. These results were also consistent with Ichikawa's result, but some regional characteristics were observed. He suggested some correlations between the earthquake mechanism and geological structure, although the data were not considered necessarily sufficient to discuss such relations, because these earthquakes were mainly those in the so-called Yodo-River seismic zone, the Wachi earthquake swarm in 1968 and others lying scattered.

Since, as mentioned in the Introduction, the Yamasaki fault is the clearest active fault along which microseismicity is very high, it would be most effective to adopt

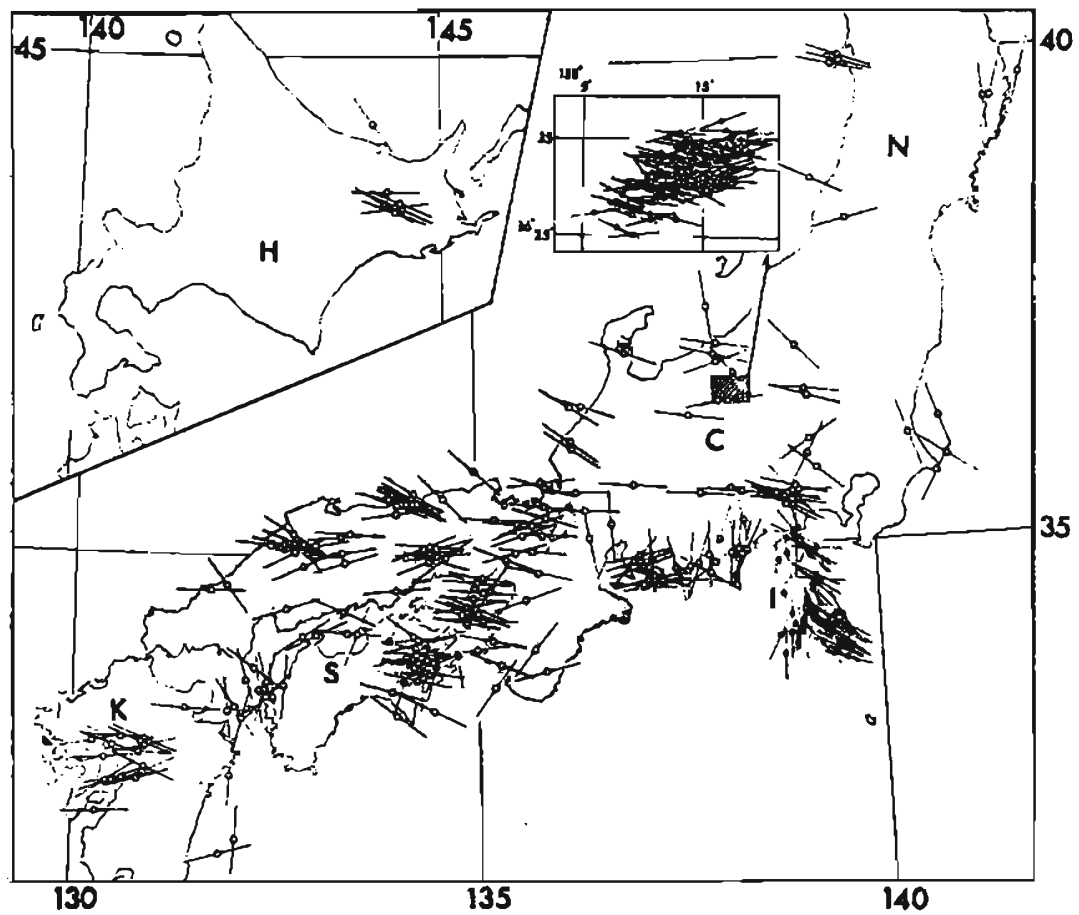

Fig. 4. Distribution of horizontal direction of the maximum pressure axis derived from large and moderate earthquakes in Southwest Japan. (after Ichikawa) 
this area in order to examine the relation between earthquake mechanisms and geological structures. For this purpose, we constructed 4 temporary stations (YZ, YS, $\mathrm{KD}, \mathrm{KZ}$ in Fig. 1) surrounding the fault, and had continued special observation for about two and half years. In this paper we shall treat with the earthquakes of magnitude range of ahout 1 to 2.5 (Table 1). Investigation of smaller earthquakes will be published in a succeeding article.

Fig. 5 shows the earthquakes used in the present analysis (large black circle), superposed on a microseismicity map from 1965 to 1968 (small dots). As shown in this figure, we classified the examined earthquakes into 8 groups, A to $\mathrm{H}$, according to the following reasons: A region corresponds to the central part of the Yamasaki fault, in which microseismicity is spacially concentrated in a narrow linear belt. On the other hand, earthquake occurrence along this line is rather intermittent, sometimes with intervals over several months. These observational facts may suggest the stickslip of a sharp strike-slip fault.

$B$ region is, in a sense, the most interesting. As shown in Fig. 3, we can observe an off-shoot fault making an acute angle with the main Yamasaki fault in this region. It is to be noted that this off-shoot fault may be the most active, left-lateral strike-slip fault, judging from the topographical evidences. Perhaps corresponding to this geological aspect, microseismicity is very high in this area.

$\mathrm{C}$ region is defined as a microseismic zone along the Harima-Nada coast line.

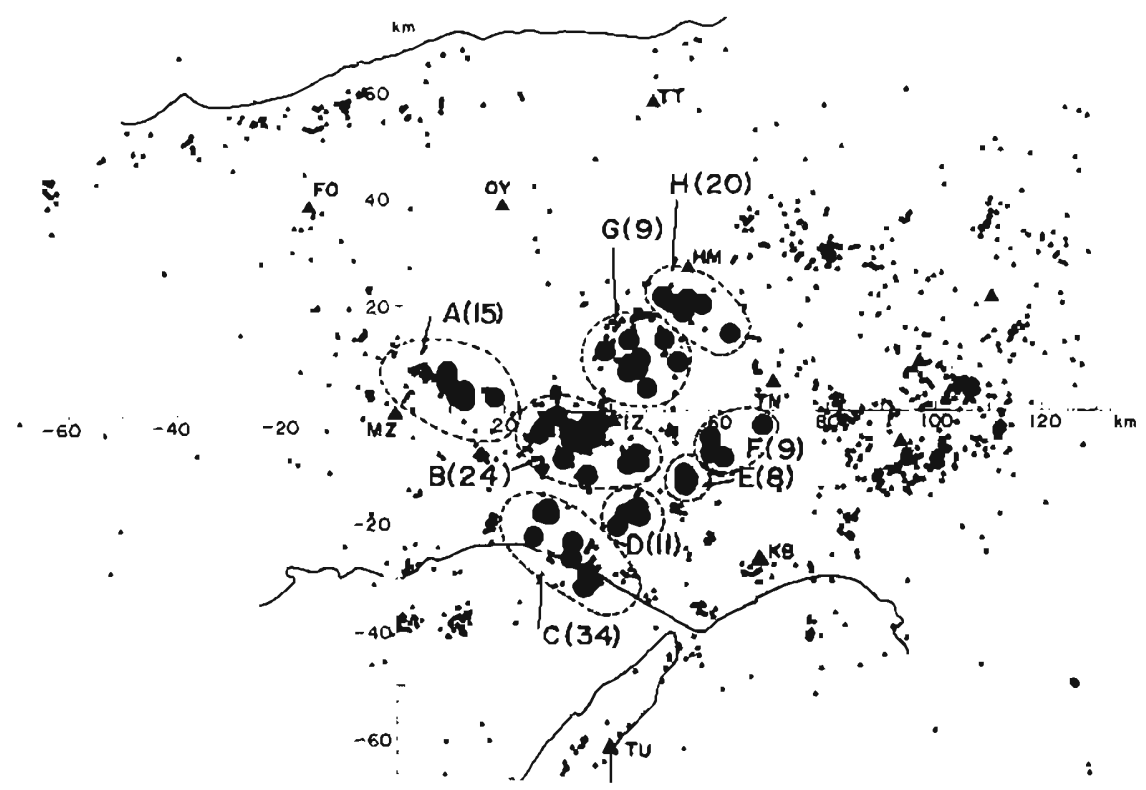

Fig. 5. Distribution of microearthquakes used in this analysis (large circles) superposed on microseismicity map. A to $H$ denote divided regions used in this analysis. Numerals in the parentheses show numbers of data. 
Tatsle 1. I.ist of microearthquakes used: I ocation of epicenter is represented by a coordinate system with the origin at $\mathrm{MZ}$ station. Azimuths of $\mathrm{P}$ and $\mathrm{T}$ axes are expressed by angles in degree measured clockwise from $\mathrm{E}$ or $\mathrm{W}$ direction, and $\mathrm{N}$ or $\mathrm{S}$ direction, respectively. $\theta$ denotes dip angles in degree. $s, t$ and $n$ in the last column represent strike-slip, thrust and normal fault types, respectively.

A Region

\begin{tabular}{|c|c|c|c|c|c|c|c|c|c|c|c|c|c|c|}
\hline \multirow{2}{*}{ No. } & \multirow{2}{*}{\multicolumn{3}{|c|}{ Date }} & \multirow[b]{2}{*}{ h } & \multirow[b]{2}{*}{$\mathrm{m}$} & \multirow[b]{2}{*}{$\begin{array}{l}\mathrm{X} \\
\mathrm{km}\end{array}$} & \multirow[b]{2}{*}{$\begin{array}{l}Y \\
\mathrm{~km} \mathrm{~m}_{\mathrm{i}}\end{array}$} & \multirow[b]{2}{*}{$\begin{array}{l}Z \\
\mathrm{~km}\end{array}$} & \multirow[b]{2}{*}{$\mathrm{M}$} & \multicolumn{2}{|l|}{$\mathrm{P}$} & \multicolumn{2}{|l|}{$\mathrm{T}$} & \multirow{2}{*}{ Type } \\
\hline & & & & & & & & & & $\mathrm{Az}$ & $\theta$ & $\mathrm{Az}$ & $\theta$ & \\
\hline 1 & 1969 & Mar. & 11 & 13 & 58 & 9.6 & 5.4 & 12 & 1.5 & & & & & \\
\hline 2 & & & 25 & 14 & 26 & 9.3 & 5.7 & 11 & 2.0 & & & & & \\
\hline 3 & & & 25 & 14 & 31 & 9.2 & 5.1 & 11 & 1.5 & & & & & \\
\hline 4 & & & 29 & 12 & 30 & 9.4 & 5.9 & 7 & 2.0 & & & & & \\
\hline 5 & & Apr. & 1 & 17 & 11 & 8.9 & 5.9 & 11 & 1.7 & & & & & \\
\hline 6 & & & 2 & 3 & 31 & 9.0 & 5.2 & 12 & 1.3 & & & & & \\
\hline 7 & & & 8 & 15 & 40 & 8.9 & 5.0 & 11 & 2.0 & & & & & \\
\hline 8 & & Sept. & 21 & 11 & 17 & 9.1 & 6.8 & 11 & 1.2 & & & & & \\
\hline 9 & 1970 & Mar. & 11 & 3 & 19 & 9.3 & 7.8 & 7 & 1.8 & $\mathrm{E}, 11 \mathrm{~N}$ & 27 & S13W & 27 & $s$ \\
\hline 10 & & & 12 & 21 & 10 & 12.6 & 4.1 & 11 & 2.5 & W $2 N$ & 7 & $S 2 W$ & 7 & $s$ \\
\hline 11 & 1971 & Jan. & 29 & 19 & 16 & 9.7 & 5.3 & 15 & 2.0 & E $9 N$ & 21 & $S 8 E$ & 3 & $s$ \\
\hline 12 & & & 29 & 21 & 10 & 9.5 & 6.0 & 15 & 1.5 & E $4 \mathrm{~S}$ & 0 & $N 4 E$ & 0 & $\mathrm{~s}$ \\
\hline 13 & & Mar. & 23 & 23 & 18 & 18.3 & 2.7 & 13 & 1.4 & E $4 \mathrm{~N}$ & 10 & $S 3 F$ & 4 & $s$ \\
\hline 14 & & May & 5 & 22 & 0 & 12.6 & 2.9 & 13 & 1.6 & W10N & 11 & N14E, & 17 & $\mathbf{s}$ \\
\hline 15 & & & 12 & 15 & 6 & 12.0 & 3.4 & 13 & 1.6 & E $9 \mathrm{~S}$ & 11 & $\mathrm{~N} 2 \mathrm{~W}$ & 46 & $t$ \\
\hline
\end{tabular}

I3 Region

\begin{tabular}{|c|c|c|c|c|c|c|c|c|c|c|c|c|c|c|}
\hline \multirow{3}{*}{ No. } & \multirow{3}{*}{ 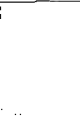 } & \multirow{3}{*}{\multicolumn{2}{|c|}{ Date }} & \multirow[b]{3}{*}{ h } & \multirow[b]{3}{*}{$m i$} & \multirow[b]{2}{*}{$x$} & \multirow[b]{2}{*}{$\mathrm{Y}$} & \multirow[b]{2}{*}{2} & \multirow{3}{*}{ M } & \multicolumn{2}{|c|}{$P$} & \multicolumn{2}{|l|}{$\mathrm{T}$} & \multirow{3}{*}{ Type } \\
\hline & & & & & & & & & & & & & & \\
\hline & & & & & & $\mathrm{km}$ & $\mathrm{km}$ & $\mathrm{km}$ & & $\mathrm{Az}$ & 10 & $\mathrm{Az}$ & $\theta$ & \\
\hline 1 & 1968 & Oct. & 23 & 14 & 40 & 45.0 & -9.4 & 14 & 2.3 & E15S & 7 & S16W & 7 & 5 \\
\hline 3 & & & 25 & 17 & 32 & 37.6 & -2.2 & 14 & 2.2 & $\mathrm{E} 40 \mathrm{~N}$ & 7 & N40W & 7 & $\mathrm{~s}$ \\
\hline 2 & & & 27 & 11 & 3 & 36.2 & -1.8 & 15 & 1.9 & W54S & 136 & N40W & 20 & $\mathbf{s}$ \\
\hline 4 & & Nov. & 7 & 6 & 40 & 31.5 & -8.6 & 12 & 1.2 & & & & & \\
\hline 5 & & Dec. & 10 & 15 & 34 & 32.8 & -5.9 & 15 & 1.0 & & & & & \\
\hline 6 & & & 10 & 21 & 30 & 33.2 & -5.2 & 15 & 1.3 & & & & & \\
\hline 7 & & & 20 & 18 & 40 & 32.6 & -6.2 & 17 & 2.1 & & & & & \\
\hline 8 & & & 21 & 20 & 21 & $27.7^{1}$ & -2.9 & 12 & 1.1 & & & & & \\
\hline 9 & 1969 & Jan. & 20 & 10 & 55 & 32.1 & -5.9 & 15 & 1.8 & W36S & 17 & N25W & 32 & $s$ \\
\hline 10 & & Mar. & 12 & 15 & 46 & 30.4 & -9.2 & 13 & 1.4 & & & & & \\
\hline 11 & & & 12 & 15 & 52 & 30.7 & -8.9 & 14 & 1.8 & E25S & 7 & S25W & 7 & s \\
\hline
\end{tabular}




\begin{tabular}{|c|c|c|c|c|c|c|c|c|c|c|c|c|c|c|}
\hline 12 & & June & 2 & 22 & 25 & 29.8 & -0.9 & 17 & 1.4 & E38N & 0 & N38W & 0 & $\mathrm{~s}$ \\
\hline 13 & & July & 25 & 13 & 32 & 42.8 & -9.8 & 12 & 2.2 & E35N & 0 & N35W & 0 & $\mathbf{s}$ \\
\hline 14 & & Oct. & 9 & 14 & 15 & 45.0 & -8.8 & 14 & 1.9 & & & & & \\
\hline 15 & & Nov. & 4 & 15 & 19 & 28.1 & -5.5 & 18 & 1.6 & E23N & 0 & $\mathrm{~N} 23 \mathrm{~W}$ & 0 & $s$ \\
\hline 16 & $: 1970$ & Apr. & 23 & 10 & 19 & 27.1 & -2.9 & 11 & - & $\mathrm{E} 34 \mathrm{~N}$ & 0 & $\mathrm{~N} 34 \mathrm{~W}$ & 0 & s \\
\hline 17 & & May & 9 & 17 & 25 & 32.6 & -5.4 & 14 & 1.7 & W31S & 24 & $\mathrm{~N} 9 \mathrm{~W}$ & 41 & s \\
\hline 18 & & & 11 & 1 & 18 & 26.7 & -3.2 & 14 & 1.6 & $\mathrm{E} 7 \mathrm{~N}$ & 0 & $N 7 W$ & 0 & $\mathrm{~s}$ \\
\hline 19 & & July & 16 & 14 & 11 & 32.2 & -3.9 & 15 & 1.6 & $\mathrm{E} 45 \mathrm{~N}$ & 0 & N45W & 0 & $s$ \\
\hline 20 & $\mid$ & Sept. & 21 & 17 & 30 & 34.7 & -3.5 & 12 & 1.8 & W37S & 31 & $\mathrm{~N} 17 \mathrm{~W}$ & 31 & $\mathbf{s}$ \\
\hline 21 & & & 24 & 9 & 45 & 34.6 & -3.6 & 14 & 2.0 & W30S & 24 & N20W & 23 & $\mathbf{s}$ \\
\hline 22 & 1971 & Feb. & 7 & 7 & 49 & 36.6 & -4.7 & 1 & 1.9 & W $5 \mathrm{~S}$ & 5 & $\mathrm{~N} 4 \mathrm{~W}$ & 5 & s \\
\hline 23 & & Mar. & 31 & 20 & 39 & 35.6 & -11.8 & 5 & 2.9 & W32S & 22 & $\mathrm{~N} 23 \mathrm{~W}$ & 22 & 5 \\
\hline 24 & & May & 8 & 8 & 27 & 34.1 & -5.4 & 10 & 1.2 & E20S & 0 & N20E & 0 & $s$ \\
\hline
\end{tabular}

\section{Region}

\begin{tabular}{|c|c|c|c|c|c|c|c|c|c|c|c|c|c|c|}
\hline \multirow[b]{2}{*}{ No. } & \multicolumn{5}{|c|}{ I } & & \multirow[b]{2}{*}{$Y_{k m}$} & \multirow[b]{2}{*}{$z$} & \multirow[b]{2}{*}{$\mathbf{M}$} & \multicolumn{2}{|l|}{$\mathrm{P}$} & \multicolumn{2}{|l|}{$\Upsilon$} & \multirow{2}{*}{ Type } \\
\hline & | & $\mathrm{D}$ & & $\mathrm{h}$ & $\mathrm{m}$ & & & & & $\mathrm{Az}$ & $\theta$ & $\mathrm{A} z$ & $\theta$ & \\
\hline 1 & 1969 & Mar. & 14 & 27 & 8 & 28.9 & -19.4 & 14 & 1.9 & & & & & \\
\hline 2 & & Apr. & 6 & 20 & 37 & 28.2 & -18.5 & 14 & 1.9 & W10S & 7 & Nlow & s & $\mathbf{s}$ \\
\hline 3 & & & 7 & 1 & 6 & 28.3 & -18.6 & 14 & 1.6 & & & & & \\
\hline 4 & & & 7 & 8 & 44 & 28.0 & -19.6 & 13 & 2.0 & & & & & \\
\hline 5 & & & 25 & 17 & 17 & 27.8 & -17.8 & 15 & 1.7 & & & & & \\
\hline 6 & 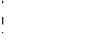 & May & 3 & 16 & 39 & 27.9 & -17.7 & 12 & 1.5 & & & & & \\
\hline 7 & & & 15 & 8 & 57 & 27.8 & -17.9 & 15 & 2.0 & & & & & \\
\hline 8 & & & 15 & 16 & 4 & 28.0 & -18.4 & 14 & 2.3 & $E 8 \mathrm{~N}$ & 7 & S 7E & & $\mathrm{s}$ \\
\hline 9 & & & 15 & 16 & 6 & 27.5 & -18.9 & 13 & 1.5 & & & & & \\
\hline 10 & $\vdots$ & & 17 & 16 & 21 & 26.3 & -18.6 & 15 & 1.9 & & & & & \\
\hline 11 & 1969 & May & 18 & 6 & 16 & 27.9 & -18.2 & 14 & 1.6 & & & & & \\
\hline 12 & & & 20 & 19 & 50 & 28.4 & -18.4 & 15 & 1.5 & & & & & i \\
\hline 13 & & & 30 & 2 & 26 & 27.9 & -18.0 & 14 & 1.3 & & & & & \\
\hline 14 & & June & 5 & 7 & 19 & 28.4 & -18.6 & 14 & 1.7 & & & & & \\
\hline 15 & & & 8 & 8 & 59 & 35.0 & -25.0 & 12 & 1.6 & & & & & \\
\hline 16 & ! & & 11 & 9 & 36 & 28.6 & -18.9 & 12 & 1.8 & & & & & : \\
\hline 17 & & & 13 & 8 & 16 & 28.3 & -19.1 & 14 & 1.8 & & & & & \\
\hline
\end{tabular}




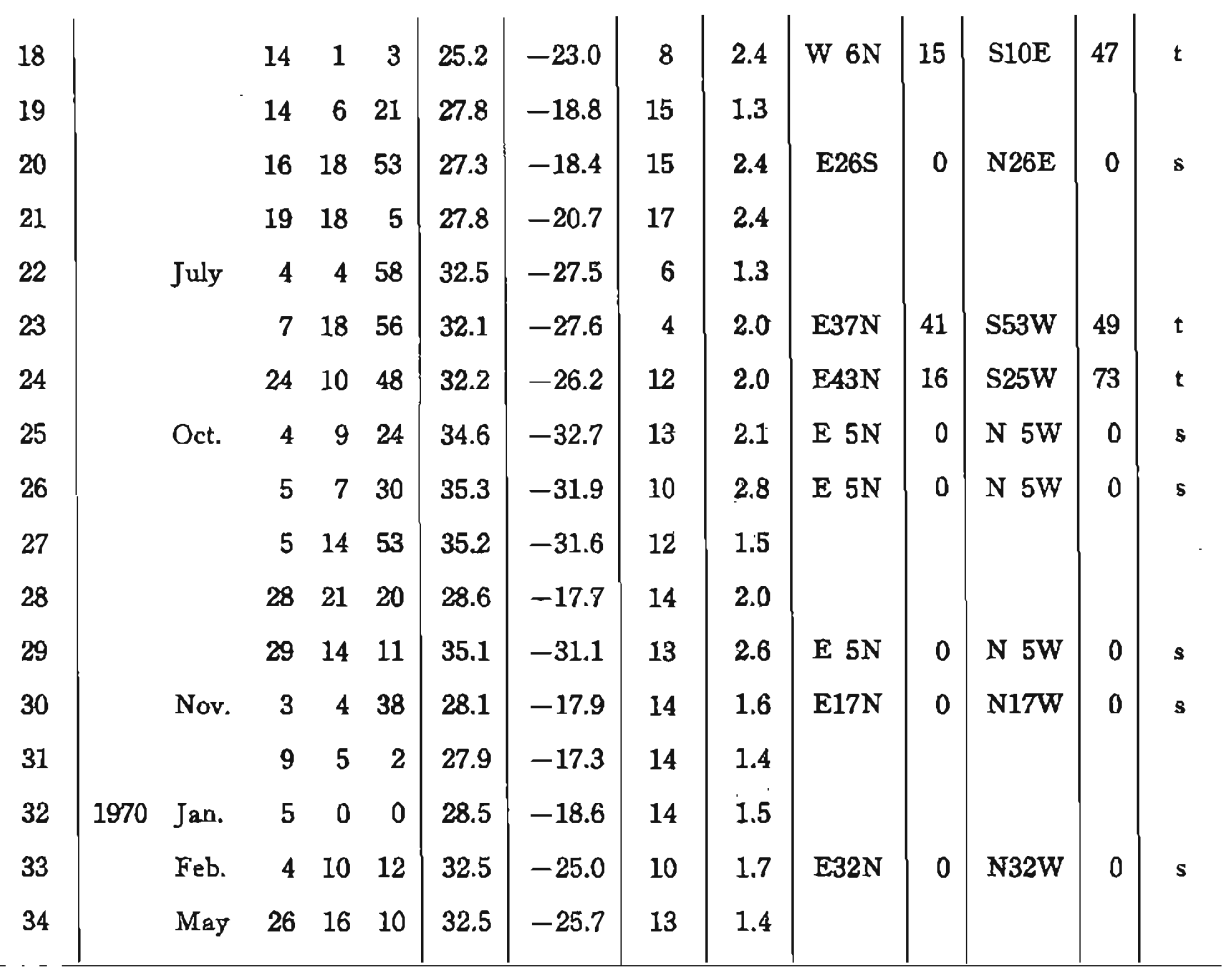

D Region

\begin{tabular}{|c|c|c|c|c|c|c|c|c|c|c|c|c|c|c|}
\hline \multirow{2}{*}{ No. } & \multirow{2}{*}{\multicolumn{3}{|c|}{ Date }} & \multirow[b]{2}{*}{ h } & \multirow[b]{2}{*}{$\mathbf{m}$} & \multirow{2}{*}{$\begin{array}{l}\mathrm{X} \\
\mathrm{km}\end{array}$} & \multirow{2}{*}{$\begin{array}{l} \\
\quad \mathrm{km} \\
\end{array}$} & \multirow{2}{*}{$\begin{array}{l}\mathrm{Z} \\
\mathrm{km}\end{array}$} & \multirow{2}{*}{$\mathbf{M}$} & \multicolumn{2}{|l|}{ P } & \multicolumn{2}{|l|}{$\mathrm{T}$} & \multirow{2}{*}{ Type } \\
\hline & & & & & & & & & & $\mathrm{Az}$ & $\theta$ & $\mathrm{Az}$ & $\theta$ & \\
\hline 1 & 1968 & Dec. & 19 & 11 & 7 & 41.4 & -21.2 & 14 & 2.2 & $\mathrm{E} 25 \mathrm{~W}$ & 0 & $\mathrm{~N} 25 \mathrm{~W}$ & 0 & 8 \\
\hline 2 & & & 26 & 5 & 35 & 41.4 & -20.6 & 14 & 1.4 & & & & & \\
\hline 3 & 1968 & Dec. & 29 & 2 & 15 & 42.4 & -21.0 & 11 & 1.6 & & & & & \\
\hline 4 & 1969 & Mar. & 4 & 3 & 42 & 42.6 & -22.3 & 4 & 2.2 & E10N & 0 & N10W & 0 & s \\
\hline 5 & 1970 & May & 12 & 19 & 56 & 42.0 & -18.9 & 15 & 1.4 & E27N & 17 & S27E & 4 & $\mathbf{s}$ \\
\hline 6 & & Dec. & 4 & 22 & 43 & 44.7 & -19.3 & 21 & 1.6 & $\mathrm{E} 20 \mathrm{~N}$ & 0 & $\mathrm{~N} 20 \mathrm{~W}$ & 0 & $\mathbf{s}$ \\
\hline 7 & & & 8 & 8 & 43 & 44.4 & -20.0 & 13 & 1.4 & & & & & \\
\hline 8 & & & 8 & 17 & 45 & 44.7 & -17.9 & 15 & 2.2 & E13N & 0 & N13W & 0 & $\mathbf{s}$ \\
\hline 9 & 1971 & Jan. & 23 & 22 & 44 & 46.6 & -18.3 & 15 & 1.3 & & & & & \\
\hline 10 & & Apr. & 13 & 8 & 18 & 44.7 & -17.7 & 13 & 1.5 & E38S & 27 & $\mathrm{~N} 22 \mathrm{E}$ & 27 & $\mathbf{s}$ \\
\hline 11 & & June & 7 & 4 & 36 & 44.1 & -18.6 & 13 & 2.7 & E15S & 0 & $\mathrm{~N} 15 \mathrm{E}$ & 0 & $\mathbf{s}$ \\
\hline
\end{tabular}


E Region

\begin{tabular}{|c|c|c|c|c|c|c|c|c|c|c|c|c|c|c|}
\hline \multirow{3}{*}{ No. } & & \multirow{2}{*}{\multicolumn{2}{|c|}{ Date }} & \multirow{3}{*}{$\mathrm{h}$} & \multirow{3}{*}{$\mathrm{m}$} & \multirow{3}{*}{$\begin{array}{c}\mathrm{X}_{\mathrm{k}} \\
\mathrm{km}\end{array}$} & \multirow{3}{*}{$\begin{array}{l}Y \\
\mathrm{~km}\end{array}$} & \multirow{3}{*}{$\begin{array}{c}Z \\
\mathrm{~km}\end{array}$} & \multirow{3}{*}{ M } & \multicolumn{2}{|l|}{ P } & \multirow{2}{*}{\multicolumn{2}{|c|}{$T$}} & \multirow{3}{*}{ Type } \\
\hline & & & & & & & & & & - & & & & \\
\hline & & & & & & & & & & & & & & \\
\hline 1 & 1969 & Mar. & 21 & 9 & 44 & 54.5 & -11.6 & 13 & 1.5 & E $2 S$ & 0 & $\mathrm{~S} 2 \mathrm{~W}$ & 29 & $s$ \\
\hline 2 & & Apr. & 16 & 21 & 1 & 54.2 & -12.4 & 12 & 1.6 & & & & & \\
\hline 3 & & & 16 & 21 & 2 & 53.9 & -12.4 & 13 & 1.7 & . & & & & \\
\hline 4 & & & 17 & 0 & 38 & 54.1 & -12.5 & 13 & 1.9 & E $8 N$ & 5 & $S 6 E$ & 20 & $s$ \\
\hline 5 & & & 17 & 0 & 46 & 53.8 & -11.7 & 12 & 1.7 & $\mathrm{E}$ & 0 & $N$ & 0 & $\mathrm{~s}$ \\
\hline 6 & & & 17 & 5 & 32 & 53.3 & -11.0 & 12 & 1.3 & & & & & \\
\hline 7 & 1970 & Sept. & 1 & 10 & 2 & 53.6 & -11.9 & 10 & 2.2 & W14N & 14 & S11W & 14 & $\mathbf{s}$ \\
\hline 8 & & Oct. & 30 & 20 & 16 & 53.1 & -13.1 & 11 & 1.1 & $\mathrm{E} 25 \mathrm{~N}$ & 0 & $\mathrm{~N} 25 \mathrm{~W}$ & 0 & s \\
\hline
\end{tabular}

F Region

\begin{tabular}{|c|c|c|c|c|c|c|c|c|c|c|c|c|c|c|}
\hline \multirow{3}{*}{ No. } & \multirow{3}{*}{\multicolumn{3}{|c|}{ Datc }} & \multirow{3}{*}{$\mathrm{b}$} & \multirow{3}{*}{$\mathrm{m}$} & \multirow{3}{*}{$\begin{array}{c}X \\
\mathrm{~km}\end{array}$} & & \multirow{3}{*}{$\begin{array}{l}\mathrm{Z} \\
\mathrm{km}\end{array}$} & \multirow{3}{*}{$M$} & \multicolumn{2}{|l|}{$\mathrm{P}$} & \multicolumn{2}{|l|}{$\mathrm{T}$} & \multirow{3}{*}{ Type } \\
\hline & & & & & & & & & & & & & & \\
\hline & & & & & & & & & & & & & & \\
\hline 1 & 1969 & June & 20 & 15 & 33 & 58.2 & -6.3 & 4 & 2.4 & W20s & 35 & N48E & 54 & $\mathrm{t}$ \\
\hline 2 & & & 20 & 16 & 7 & 58.1 & -7.2 & 21 & 2.6 & $W 4 S$ & 5 & N85E & 85 & $\mathrm{t}$ \\
\hline 3 & & & 20 & 20 & 13 & 58.2 & -5.4 & 14 & 2.0 & W 9S & 17 & N14E & 53 & $\mathrm{t}$ \\
\hline 4 & & & 20 & 20 & 54 & 58.0 & -6.1 & 14 & 1.7 & & & & & \\
\hline 5 & & & 22 & 6 & 58 & 58.6 & -5.6 & 14 & 2.3 & E $5 S$ & 10 & N85W & 80 & $\mathrm{t}$ \\
\hline 6 & & & 28 & 18 & 6 & 59.1 & -6.2 & 14 & 2.3 & & & & & \\
\hline 7 & 1969 & July & 12 & 6 & 14 & 60.7 & -8.5 & 8 & 1.5 & & & & & \\
\hline 8 & & & 12 & 6 & 15 & 58.2 & -5.5 & 13 & 1.4 & & & & & \\
\hline 9 & 1970 & June & 17 & 19 & 42 & 68.0 & -2.5 & 13 & 1.7 & & & & & \\
\hline
\end{tabular}

G Region

\begin{tabular}{|c|c|c|c|c|c|c|c|c|c|c|c|c|c|c|}
\hline \multirow{2}{*}{ No. } & \multirow{2}{*}{\multicolumn{3}{|c|}{ Date }} & \multirow[b]{2}{*}{$\mathrm{h}$} & \multirow[b]{2}{*}{$\mathrm{m}$} & \multirow{2}{*}{$\begin{array}{c}\mathrm{X} \\
\mathrm{km}\end{array}$} & \multirow{2}{*}{$\begin{array}{l}\mathrm{Y} \\
\mathrm{km}\end{array}$} & \multirow{2}{*}{$\begin{array}{l}Z \\
\mathrm{~km}\end{array}$} & \multirow{2}{*}{$M$} & \multicolumn{2}{|l|}{$P$} & \multicolumn{2}{|l|}{$\mathrm{T}$} & \multirow{2}{*}{ Typ } \\
\hline & & & & & & & & & & $\mathrm{Az}$ & $\theta$ & $\mathrm{Az}$ & $\theta$ & \\
\hline 1 & 1970 & Mar. & 15 & 6 & 7 & 43.4 & 13.2 & 7 & 2.0 & E17N & 45 & $N 6 E$ & 25 & $n$ \\
\hline 2 & & Apr. & 3 & 10 & 2 & 42.9 & 7.5 & 11 & - & $\mathrm{E} 15 \mathrm{~N}$ & 0 & $\mathrm{~N} 15 \mathrm{~W}$ & 0 & $\mathrm{~s}$ \\
\hline 3 & & June & 5 & 16 & 39 & 43.2 & 8.3 & 10 & 1.5 & W21S & 5 & N60E & 85 & $\mathrm{t}$ \\
\hline 4 & & Oct. & 5 & 17 & 47 & 39.2 & 11.5 & 6 & 1.6 & E26S & 0 & N26E & 0 & $\mathbf{s}$ \\
\hline 5 & 1971 & Jan. & 2 & 19 & 46 & 46.3 & 4.5 & 2 & 1.8 & $E$ & 43 & $\mathrm{~N} 2 \mathrm{~W}$ & 47 & t \\
\hline
\end{tabular}




\begin{tabular}{r|rrrr|r|r|r|r|r|r|r|r|r}
6 & Mar. & 5 & 18 & 46 & 50.1 & 13.4 & 25 & 1.4 & W32N & 20 & S24E & 67 & $\mathrm{t}$ \\
7 & Mar. & 19 & 4 & 27 & 44.2 & 8.1 & 13 & 1.1 & W17S & 8 & N17W & 8 & $\mathrm{~s}$ \\
8 & Apr. & 1 & 16 & 2 & 52.7 & 9.4 & 17 & 2.3 & W20S & 7 & S20E & 7 & $\mathrm{~s}$ \\
9 & & 3 & 1 & 53 & 45.5 & 10.0 & 11 & 2.0 & E17N & 3 & S17E & 3 & $\mathrm{~s}$ \\
\hline
\end{tabular}

H Region

\begin{tabular}{|c|c|c|c|c|c|c|c|c|c|c|c|c|c|c|}
\hline \multirow{2}{*}{ No. } & \multirow[t]{2}{*}{1} & \multirow{2}{*}{\multicolumn{2}{|c|}{ Date }} & \multirow[b]{2}{*}{$\mathrm{h}$} & \multirow[b]{2}{*}{$\mathrm{m}$} & \multirow{2}{*}{$\begin{array}{c}X \\
\mathrm{~km}\end{array}$} & \multirow{2}{*}{$\begin{array}{l}\mathrm{Y} \\
\mathrm{rm}\end{array}$} & \multirow{2}{*}{$\begin{array}{l}Z \\
\mathrm{~km}\end{array}$} & \multirow{2}{*}{$\mathrm{M}$} & \multicolumn{2}{|l|}{$\mathbf{P}$} & \multicolumn{2}{|l|}{$T$} & \multirow{2}{*}{ Type } \\
\hline & & & & & & & & & & $\mathrm{Az}$ & $\theta$ & $\mathrm{Az}$ & $\theta$ & \\
\hline 1 & 1968 & Aug. & 13 & 23 & 57 & 47.2 & 24.8 & 11 & - & & & & & \\
\hline 2 & & & 17 & 13 & 13 & 48.0 & 23.8 & 11 & 2.3 & $\mathrm{E} 15 \mathrm{~N}$ & 0 & N15W & 0 & s \\
\hline 3 & & & 15 & 18 & 14 & 47.6 & 24.4 & 11 & - & & & & & \\
\hline 4 & & Oct. & 5 & 3 & 11 & 54.9 & 20.3 & 7 & 1.7 & & & & & \\
\hline 5 & & & 14 & 11 & 25 & 62.0 & 14.7 & 12 & 2.0 & W84N & 83 & $\mathrm{~S} 6 \mathrm{E}$ & 12 & $n$ \\
\hline 6 & 1969 & Mar. & 3 & 7 & 13 & 61.3 & 15.5 & 7 & 1.5 & & & & & \\
\hline 7 & & & 26 & 18 & 37 & 53.5 & 18.7 & 14 & 2.3 & W10S & 7 & $\mathrm{~N} 8 \mathrm{~W}$ & 7 & s \\
\hline 8 & & July & 25 & 13 & 33 & 54.3 & 21.2 & 3 & 2.1 & F. $6 \mathrm{~S}$ & 1 & S $5 W$ & 65 & $\mathrm{t}$ \\
\hline 9 & & & 31 & 10 & 48 & 55.9 & 20.2 & 7 & 1.2 & & & & & \\
\hline 10 & & Aug. & 24 & 20 & 8 & 55.8 & 20.1 & 7 & 1.6 & & & & & \\
\hline 11 & & Dec. & 27 & 7 & 9 & 52.7 & 19.9 & 14 & 1.5 & $\mathrm{E} 15 \mathrm{~N}$ & 7 & N16W & 7 & $\mathbf{s}$ \\
\hline 12 & 1970 & Sept. & 30 & 17 & 22 & 53.2 & 22.8 & 5 & 1.3 & & & & & \\
\hline 13 & & Dec. & 22 & 22 & 34 & 51.2 & 20.9 & 12 & 1.4 & E $7 \mathrm{~N}$ & 8 & $S 2 E$ & 33 & $\mathrm{~s}$ \\
\hline 14 & 1971 & Jan. & 6 & 8 & 4 & 69.0 & 12.0 & 6 & 2.4 & & & & & \\
\hline 15 & & Mar. & 23 & 11 & 19 & 49.7 & 21.5 & 10 & 1.9 & $\mathrm{E} 68 \mathrm{~S}$ & 2 & N45E & 25 & s \\
\hline 16 & & & 23 & 11 & 21 & 49.3 & 21.1 & 9 & 2.4 & W32S & 55 & $\mathrm{~N} 58 \mathrm{E}$ & 45 & $\mathrm{n}$ \\
\hline 17 & & & 25 & 6 & 19 & 56.9 & 20.0 & 3 & 1.4 & $\mathrm{E}$ & 0 & $\mathrm{~N}$ & 0 & s \\
\hline 18 & & Apr. & 7 & 21 & 49 & 68.3 & 11.8 & 6 & 1.3 & & & & & \\
\hline 19 & & May & 3 & 22 & 33 & 69.6 & 11.7 & 7 & 1.2 & & & & & \\
\hline 20 & & June & 17 & 10 & 44 & 68.8 & 12.1 & 10 & 1.2 & & & & & \\
\hline
\end{tabular}


According to Huzita et al. ${ }^{11}$, this coast line itself is supposed a buried fault which may have the same nature as the Yamasaki fault.

$D$ region is a cluster of microearthquakes. It is interesting, as seen in Table 1, that microearthquake activity has stationarily continued in this quite small area.

$\mathrm{E}$ and $\mathrm{F}$ groups are two earthquake swarms which occurred in very small domains. They must be classified into a different kind from $A, B, C$ and $D$ groups, because the latter four groups may be stationary activity along the active faults, but the occurrences of the former two are not considered stationary in space and time.

$G$ region corresponds to a belt-like distribution of epicenter nearly in the direction connecting IZ and HM (Fig. 5), which makes a conjugate direction with the Yamasaki fault. In Fig. 3, a fault is designated along this line, but this fault, if existing, may not be such a long and clear one as the Yamasaki. Accordingly, we are interested in examining mechanisms of microearthquakes of which the linear arrangement of
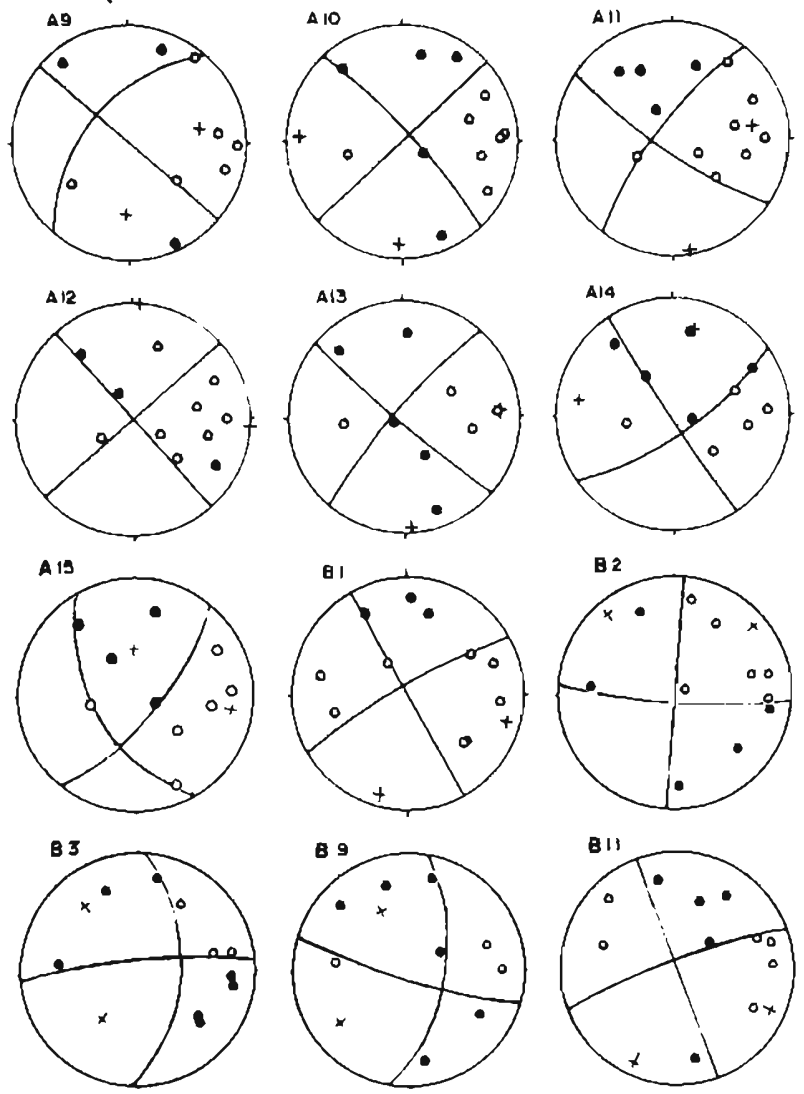

Fig. 6. Fault plane solutions by the Wulf's net projection on the upper hemisphere. Numerals attached to each figure correspond to list numbers in Table 1 . White and black circles represent pull and push motions, respectively. Crosses show $P$ and $T$ Axes. 

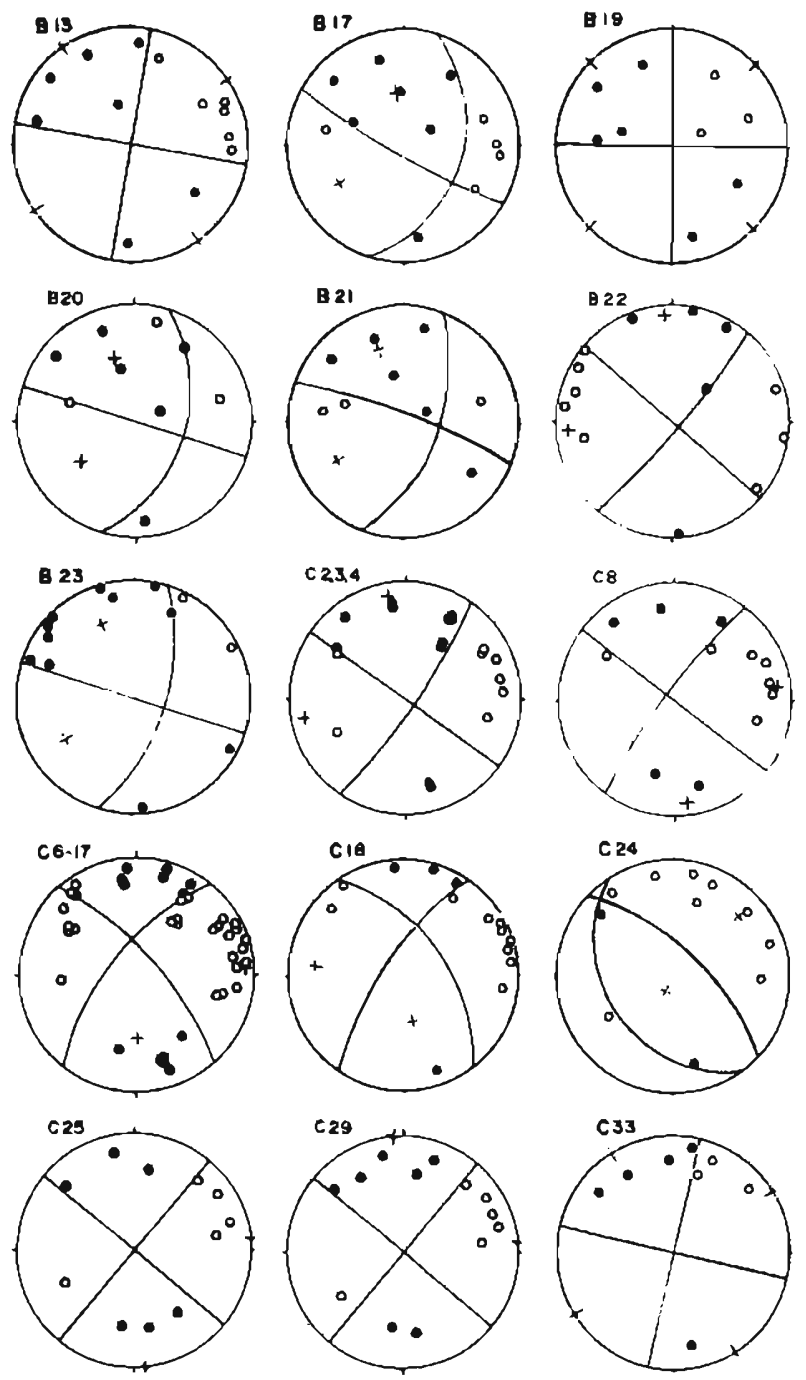

Fig. 6 (Continued) 

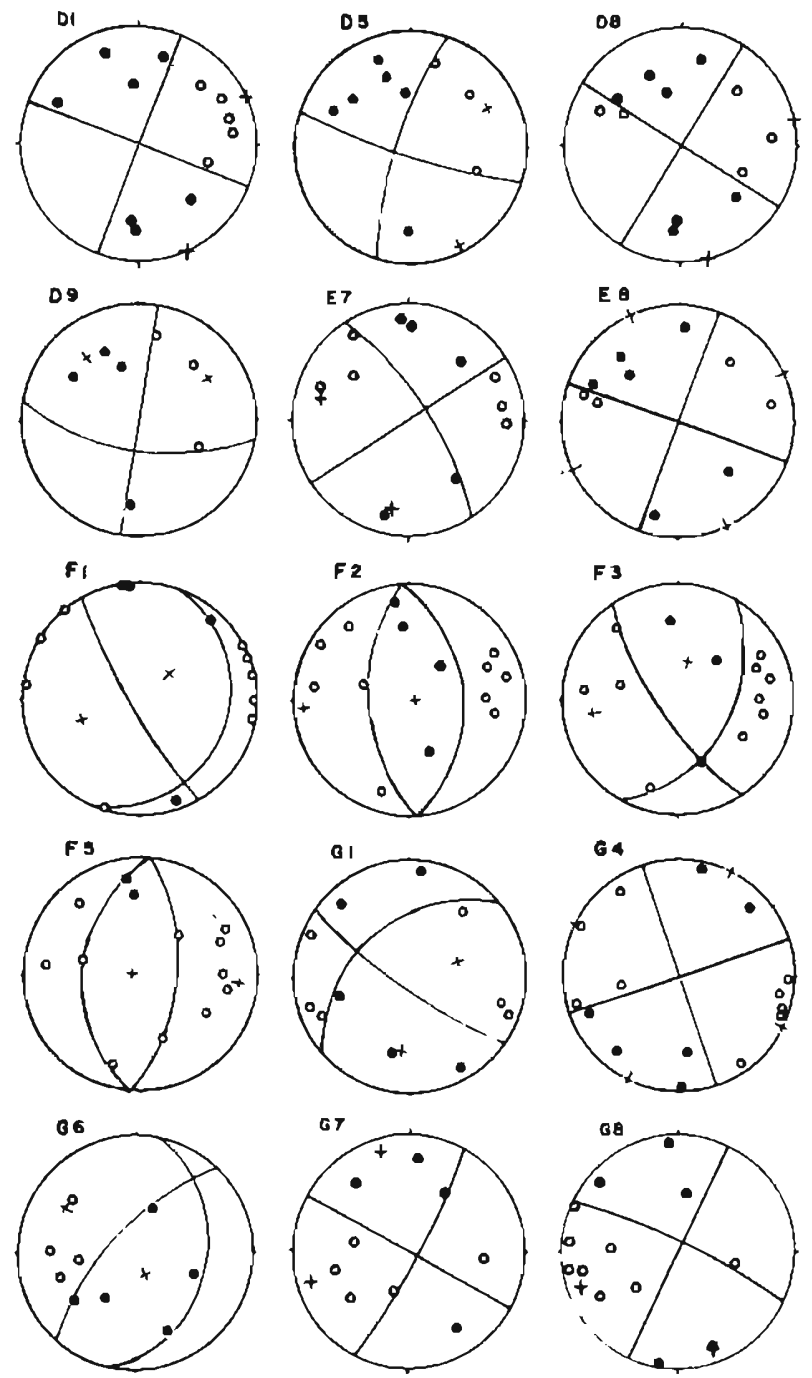

Fig. 6 (Continued) 

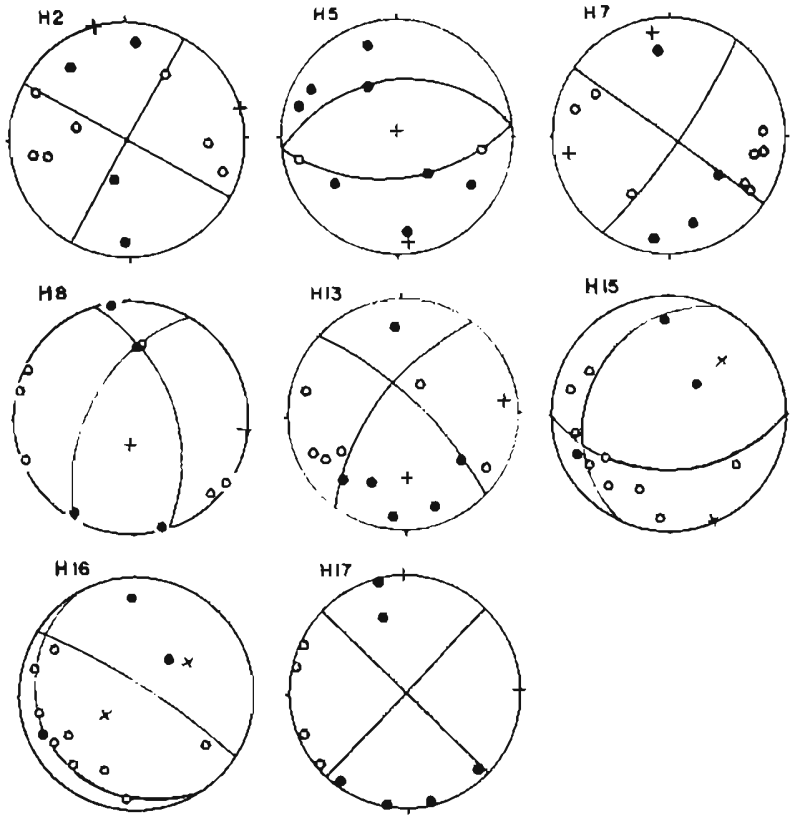

Fig. 6 (Continued)

epicenters is recognized, but corresponding geological evidences are not so clear. $\mathrm{H}$ region is considered an extension of $\mathrm{G}$ region, but a part of $\mathrm{H}$ region may extend southeastwards through TN station (Fig. 5). Along this extension a linear arrangement of epicenters are also observed, but no fault has been reported.

Table 1 shows a list of microearthquakes in $\mathrm{A}$ to $\mathrm{H}$ regions. The data used in the present analysis were selected in the period from Sept., 1968 to June, 1971, under a criterion that observations were made at more than 4 stations of the Tottori and Abuyama networks (Fig. 1). The analyses were made by the same procedures as Nishida ${ }^{12)}$ cited above, namely one is determining fault plane solutions of the respective earthquakes and the other derivation of smoothed radiation patterns in the respective regions. In this Section, we shall show the fault plane solutions and mention their characteristics.

\section{A region: Central part of the Yamasaki fault}

7 earthquakes out of 15 examples in this region could be used to determine fault plane solutions, and are shown in Fig. 6, A9 to A15. In these figures as well as all others, the Wulff's net projection on the upper hemisphere was applied. Needless to say, in many cases of analyses of microearthquakes, it is pretty difficult to exactly determine the nodal planes owing to insufficient numbers of observation and also to inadequate distribution of stations. Therefore, we adopted the following principle to proceed our analysis, namely four-quadrant type with vertical null vector is primarily 


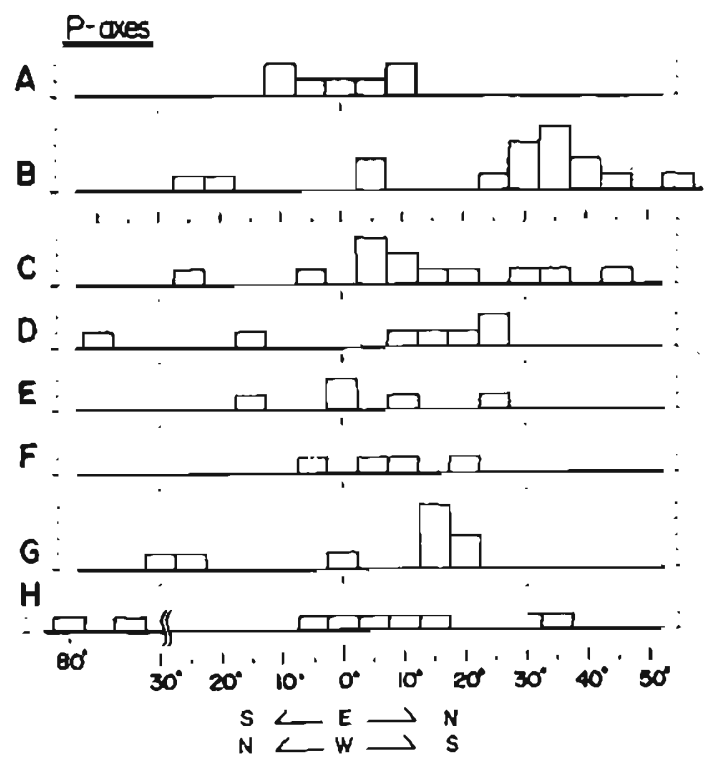

Fig. 7 (a) Frequency distribution of horizontal direction of $\mathrm{P}$ axis. Ordinate shows numbers.

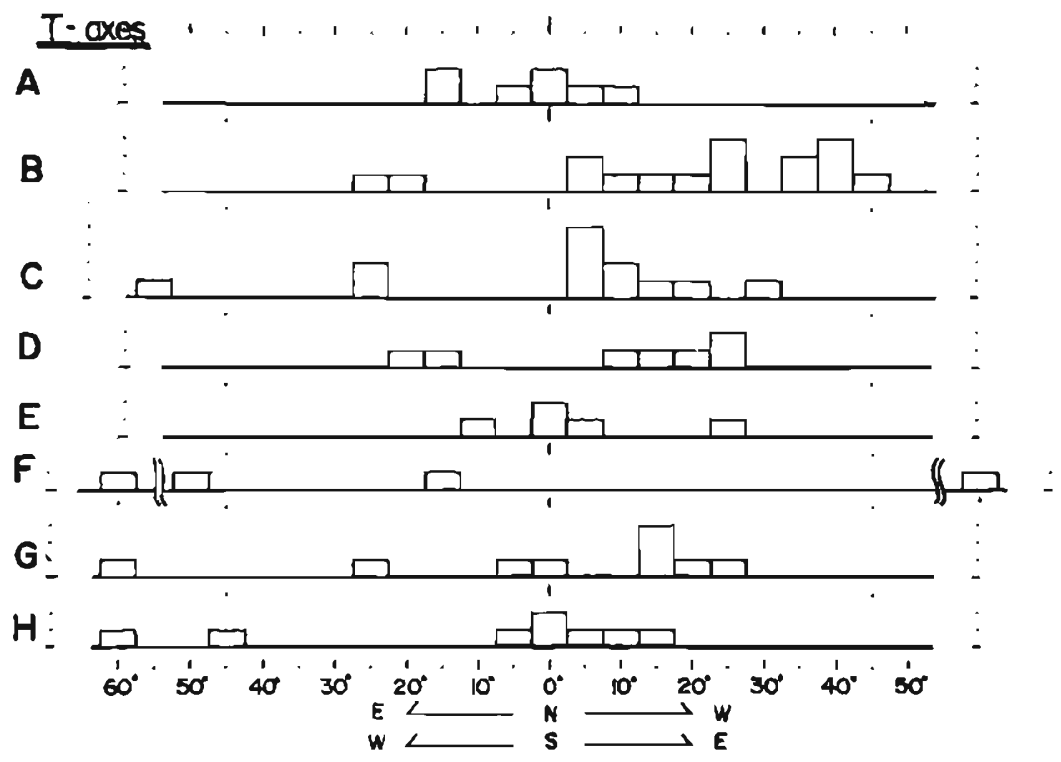

Fig. 7 (b) Frequency distriburion of horizontal direction of $\mathrm{T}$ axis. 


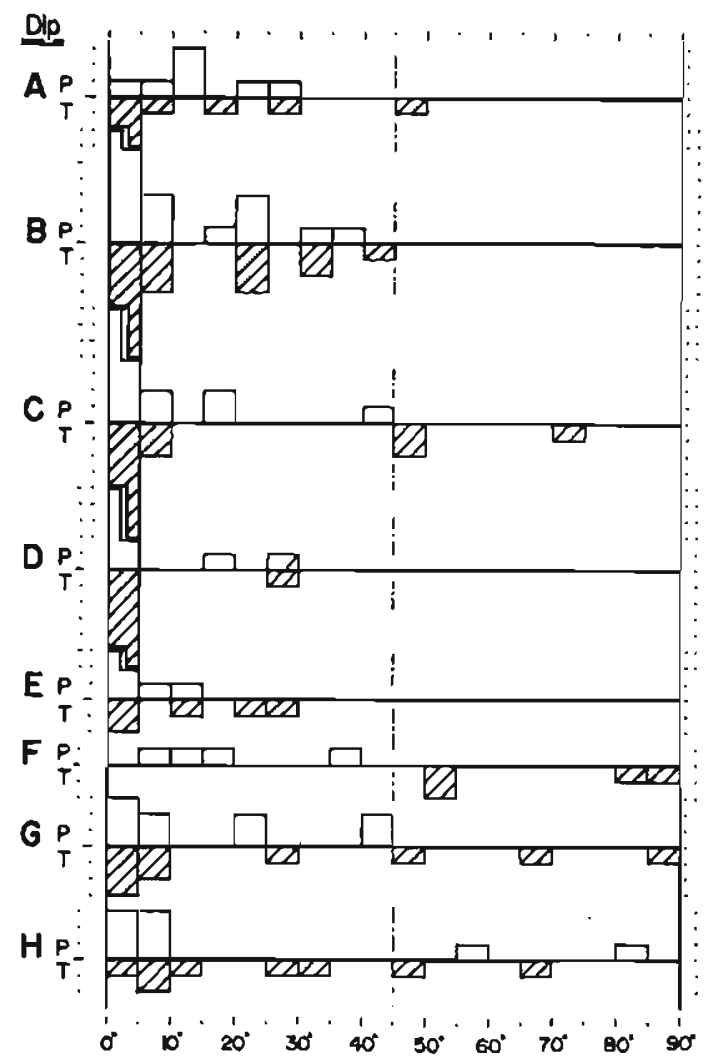

Fig. 7 (c) Frequency distriburions of dip angles of $\mathrm{P}$ and $\mathrm{T}$ axes.

employed in the northwestern Kinki area, while referring to various results so far published.

As shown in Fig. 6 and in the last three columns of Table 1, 6 examples, A9 to A14, are classified into strike-slip fault type and one, A15, into a thrust fault type. Fig. 7 (a), (b) and (c) represent frequency distributions, in respective regions, of directions of the maximum pressure $(\mathrm{P})$ and the minimum pressure $(\mathrm{T})$ axes, and dips of both axes, respectively. We can recognize that $\mathrm{P}$ and $\mathrm{T}$ axes in $\mathrm{A}$ region are concentrated in E-W and N-S directions, respectively, and dips of both axes are not so large. If, from a geological point of view, we assume one nodal plane oriented in a direction from 2nd quadrant to 4 th (nearly NW-SE) as the fault plane, those directions measured clockwise from E-direction are as follows:

$41,47,34,49,41,57,61$ (deg.).

The mean value is $E 47^{\circ} \mathrm{S}$, excepting the last one of thrust fault type.

\section{$B$ Region: Area surrounding the off-shoot fault of the Yumasaki fault}

As seen in Table 1, occurrence of microearthquakes is stationary in this area. Fault plane solutions were determined for 17 earthquakes out of 24 . The most re- 
markable nature of these earthquakes is that all examples are of strike-slip type, but that $\mathrm{P}$ and $\mathrm{T}$ axes largely deviate counterclockwise from $\mathrm{E}-\mathrm{W}$ and $\mathrm{N}-\mathrm{S}$, respectively. Deviations of both axes amount to about $30^{\circ}$, as seen in Fig. 7. This suggests that there is a clear difference in tectonic stress or orientation of the main fracture plane between $A$ and $B$ regions. Considered geologically, it seems to be more plausible to adopt the latter explanation. If we show the azimuths of the supposed fault planes in the same manner as in A Region, the result is as follows:

$$
61,4,-3,22,70,7,8,22,11,30,38,6,18.21,41,17,65 \text { (deg.). }
$$

The mean value is $\mathrm{E} 26^{\circ} \mathrm{S}$ for all examples, and $\mathrm{E} 17^{\circ} \mathrm{S}$ when 3 extraordinary ones (61, $70,65)$ are omitted. The latter value differs about $30^{\circ}$ from the mean value in A region.

\section{Region: Area of a supposed fault along the Hurima-Nada coast line}

Sometimes relatively large earthquakes $(M>3)$ occur in this region. Two of 34 earthquakes treated in the present analysis were previously analysed by Nishida ${ }^{13}$ ). Since some earthquake swarms are included in this region, as shown in Table 1, some earthquakes were superimposed, giving one fault plane solution. Generally speaking, the strike-slip fault type is prevailing also in this region. Although three earthquakes showed clear dip-slip type indications, these belong to the same swarm and therefore are not considered as being the general tendency of this region. $P$ and $T$ axes of strike-slip type earthquakes lie nearly in the middle of $\mathrm{A}$ and $\mathrm{B}$ regions, and dip angles of both axes are quite small. Strikes of the supposed fault planes are given in the same manner;

$$
35,37,48,57,71,71,28,27,40,40,40,13 \text { (deg.). }
$$

These give a mean value of $\mathrm{E} 41^{\circ} \mathrm{S}$.

\section{$D$ Region:}

Earthquakes in $\mathrm{D}$ region are situated geographically in an extension of the Yamasaki fault, and show stationary occurrence. Therefore, it may be better to include this group in $\mathrm{A}$ or $\mathrm{B}$ groups. 8 fault plane solutions were obtained, and the resulting $\mathrm{P}$ and $\mathrm{T}$ axes are both alike to that of $\mathrm{C}$ region. The mean strike of the supposed fault planes is $\mathrm{E} 34^{\circ} \mathrm{S}$.

\section{$E$ and $F$ Regions:}

Earthquakes in these two regions are two earthquake swarms which are situated at an end of the Yamaxaki fault and both swarms occurred within a very short time interval of about one month. Therefore, it seems natural that earthquakes in each group have nearly the same mechanisms. It is to be noted, however, that the last 2 earthquakes in $\mathrm{E}$ group have the same mechanisms as the other 6 in spite of time interval of one and half years between the former and the latter groups. The mean strike of the supposed fault planes is $\mathrm{E} 42^{\circ} \mathrm{S}$ in $\mathrm{E}$ region. Mechanisms of 4 earthquakes in $F$ region are of clear dip-slip type.

\section{$G$ and $H$ Regions: Microseismic belt in IZ-HM direclion}

In $G$ region it was possible to determine fault plane solutions for 9 earthquakes. 

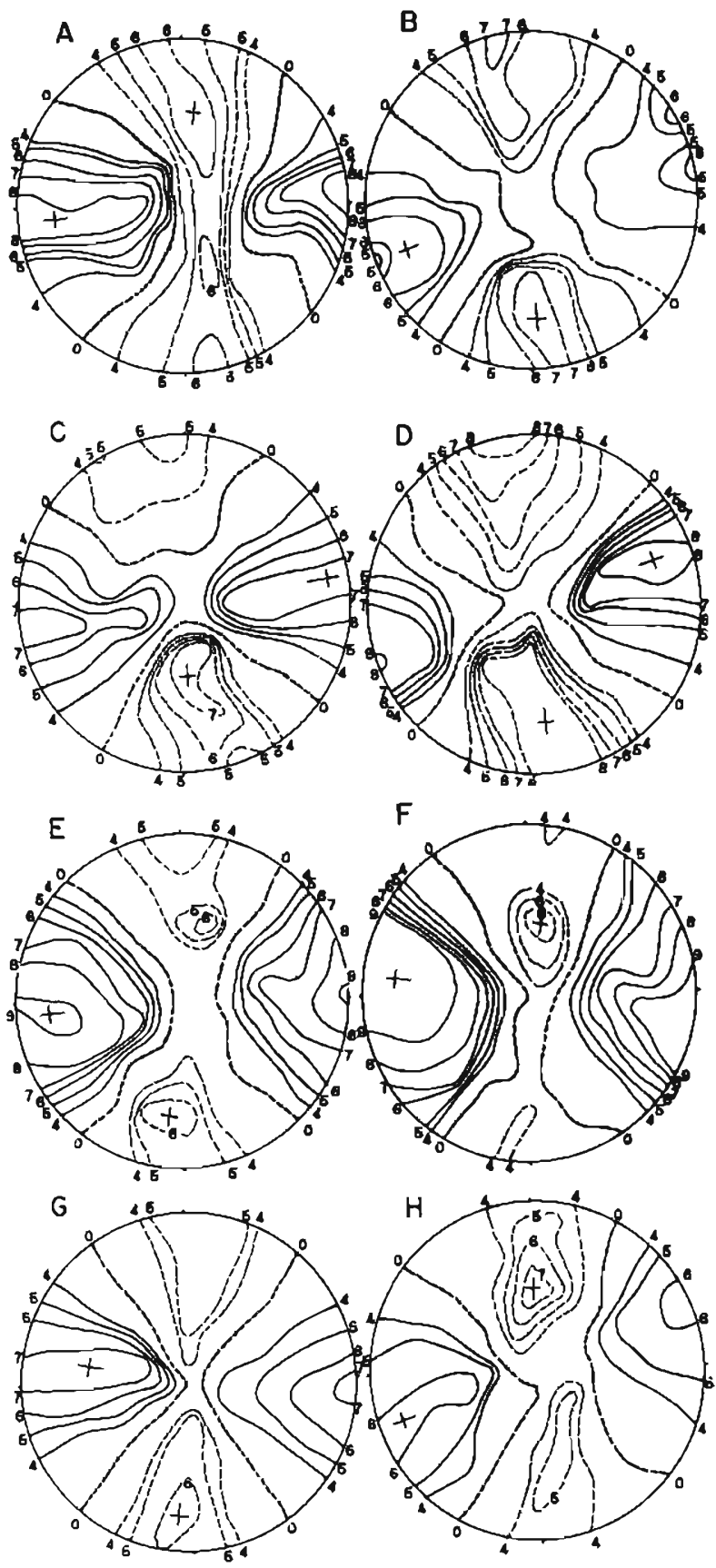

Fig. 8. Smoothed radiation pattern in each region. 
It is very interesting that these solutions include various types, strike-slip, thrust and even normal fault type. The 9 examples consist of 5 strike-slip types, 3 thrust fault types and one normal fault type. This situation is the same as in $\mathrm{H}$ region, which contains six strike-slip types, one thrust fault type and two normal fault types. Namely, in $\mathrm{G}$ and $\mathrm{H}$ regions, strike-slip types and dip-slip (thrust and normal) types occur with nearly the same frequency. This property in these two regions is quite different from A to E regions which are along the clear strike-slip fault.

\section{Smoothed radiation patterns inthe respective regions}

Analysis by smoothed radiation pattern is an effective method to estimate the general tendency of tectonic stress acting in any seismic zone. Nishida ${ }^{14)}$ divided the northwestern Kinki area into several blocks, and applied this method in order to detect the tectonic stress in each block. In the previous Section, we examined mechanisms of microearthquakes in several regions which are divided more microscopically than Nishida's blocks, and ascertained that fault plane solutions show properties characteristic in the respective regions. We shall next examine the smoothed radiation patterns in these regions by the same method as used by Nishida. In the calculation all the data in Table 1 were used.

Fig. 8 shows results calculated in each region, and Fig. 9 represents the overall pattern. In each figure, the full and dotted lines denote pull and push zones, respectively. It is striking that the overall pattern shows a beautiful pattern of the four-quadrant type with $\mathrm{P}$ and $\mathrm{T}$ axes horizontally oriented in $\mathrm{E}-\mathrm{W}$ and $\mathrm{N}-\mathrm{S}$, respectively. This result is completely consistent with various investigations in this area, as cited earlier. By more precise examination of each region in Fig. 8, however, we can observe

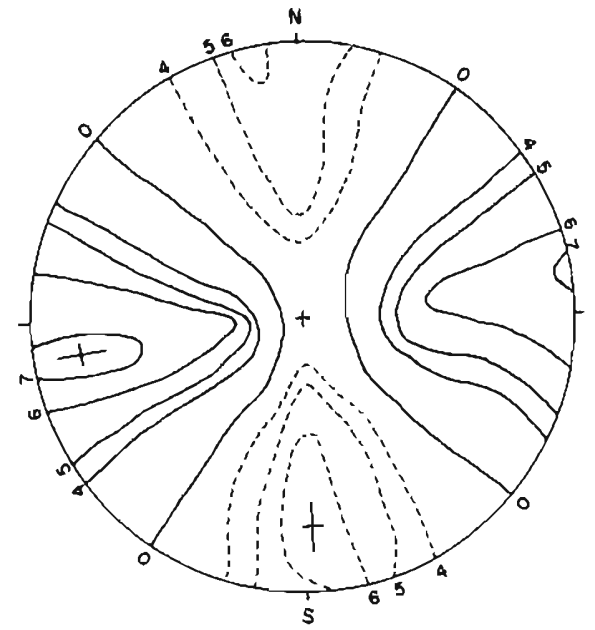

Fig. 9. Overall representation of smoothed radiation pattern summing up all regions. some differences in patterns from region to region; for instance the pattern in $B$ region is clearly different from that of $\mathrm{A}$ region. This is natural when considering the fault plane solutions in both regions. It seems particularly interesting that the smoothed radiation pattern in $G$ region shows an ordinary four-quadrant type which is nearly the same as in other regions, in spite of the fact that fault plane solutions in $G$ region contain various fault types. This may be an accidental result brought about by mixing of various types.

Accordingly, these results regarding the smoothed radiation pattern suggest that this method is useful to estimate the general tendency of tectonic stress acting 
over a relatively wide area, but we must be careful if we use this method in the case when the numbers of observations and stations are insufficient, and distribution of stations are not uniform.

\section{Some discussions}

\section{Focal depth distribution and earthquake mechanism}

Fig. 10 shows focal depth distribution of earthquakes used in each region. In A to $C$ regions, almost all earthquakes are concentrated in a depth range of 10 to $15 \mathrm{~km}$. This distribution, which is the general tendency in the northwestern Kinki area, is particularly remarkable in such strike-slip fault areas as the Yamasaki15). On the other hand, the type of distribution is different in $G$ and $H$ regions, in which eathquakes occur nearly equally in any depth down to $15 \mathrm{~km}$. This type is seen, for instance, in the aftershock area of the Tottori earthquake in 1943. It is an interesting problem whether or not the difference in focal depth distribution has any relation to the difference

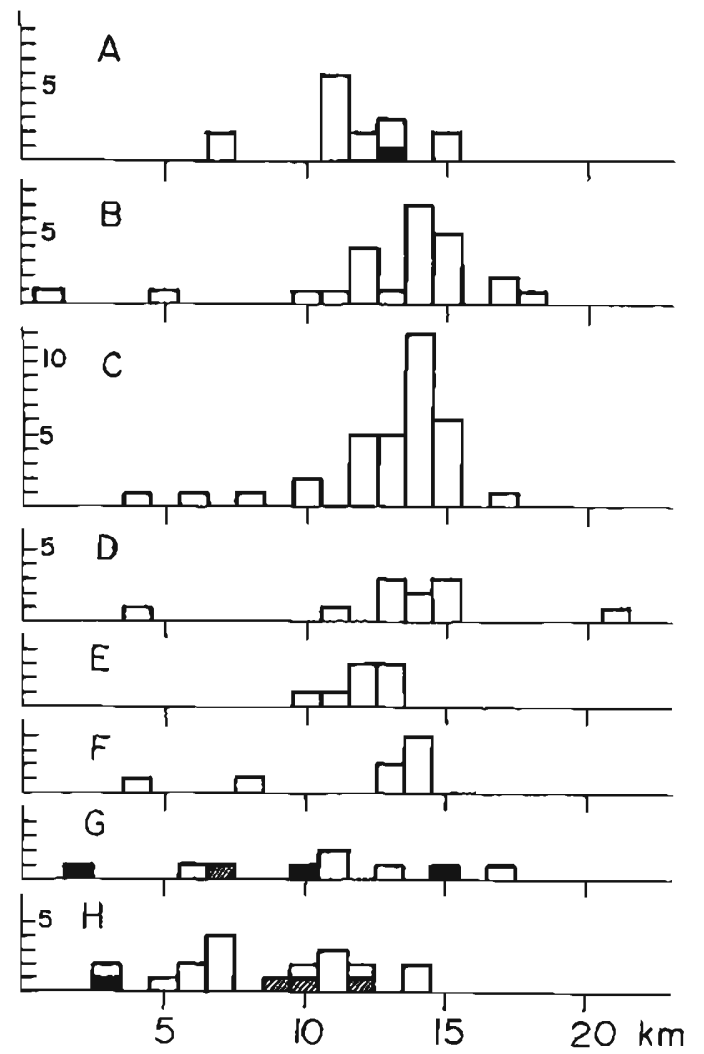

Fig. 10. Focal depth distribution in each region. Ordinate shows numbers. White, black and hatched parts represent strike-slip, thrust and normal fault types, respectively. 
in types of fault plane solutions. Although this problem needs more investigations in future, we might say that there is a certain tectonic difference between the $\mathrm{A}$ to $\mathrm{C}$ and the $\mathrm{G}$ and $\mathrm{H}$ regions.

In Fig. 10, white, tlack and hatched parts represent strikeslip, thrust and normal fault types, respectively. No correlation is observed between the fault type and the focal depth.

\section{Fault and mechanism of microearthquake}

We showed in the previous two Sections that the majority of microearthquakes ( $\mathrm{M}=1 \sim 2.5$ ) in the northwestern part of Kinki have mechanisms of strike.slip type supposed to be caused by horizonta! and F. W compression. By microscopic examinations, however, there were clear differences in focal mechanisms from region to region, and we supposed that it is caused by the difference of geological structures in the respective regions. In the following we shall discuss in some detail the relation between mechanisms and geological structures.

In $A$ region which is situated at central part of the Yamasaki fault extending over $80 \mathrm{~km}$, all earthquakes, with one exception, showed strike-slip type of which $\mathrm{P}$ and $\mathrm{T}$ axes were nearly E-W and N-S direction. This nature has been considered as the representative of earthquake mechanisms in the northwestern Kinki area. We assumed, in Section 2, one nodal plane to be the fault plane from a geological point of view. The mean strike of these nodal planes was $\mathrm{E} 47^{\circ} \mathrm{S}-\mathrm{W} 47^{\circ} \mathrm{N}$. On the other hand, the strike of the Yamasaki fault observed on the earth's surface is nearly $\mathrm{E} 30^{\circ} \mathrm{S}-\mathrm{W} 30^{\circ} \mathrm{N}$. If this postulation that one nodal plane is a real fracture surface is correct, then there is a difference of about $15^{\circ}$ in the fault trend on the surface. This fact night suggest that real fracture occurs en echelon to the fault, or the fault trend observed on the surface is just the superficial phenomenon.

$B, C, D$ and $E$ regions are understood to have fundamentally the same tectonic conditions as in A region. The mean strike of the "supposed fault planes" derived from fault plane solutions in these regions are as follow's:
$B$ region: $\mathrm{E} 17^{\circ} \mathrm{S}-\mathrm{W} 17^{\circ} \mathrm{N}$
C region: $\mathrm{E} 41^{\circ} \mathrm{S}-\mathrm{W} 41^{\circ} \mathrm{N}$
D region: $\mathrm{E} 34^{\circ} \mathrm{S}-\mathrm{W} 34^{\circ} \mathrm{N}$
$\mathrm{E}$ region: $\mathrm{E} 42^{\circ} \mathrm{S}-\mathrm{W} 42^{\circ} \mathrm{N}$.

It is remarkable that the value in $\mathrm{B}$ region definitely differs from the other 4 regions, $\mathrm{A}$, $\mathrm{C}, \mathrm{D}$ and $\mathrm{E}$. As mentioned earlier, in $\mathrm{B}$ region there is an active off-shoot of the main Yamasaki fault. The strike of the off-shoot measured geographically is E $16^{\circ} \mathrm{S}-\mathrm{W} 16^{\circ} \mathrm{N}$ which completely coincides with $\mathrm{E} 17^{\circ} \mathrm{S}-\mathrm{W} 17^{\circ} \mathrm{N}$ described above. On the other hand, the fault strikes in $C$ and $D$ regions are considered parallel to the Yamasaki fault, and trends of the supposed fault planes are also alike to that $A$ region. These facts seem very interesting; namely, along the main part of strike-slip faults the strike of fault estimated geologically makes some acute angles with the corresponding fault strike derived from the fault plane solution. On the contrary, both values mentioned above 
are in good accordance along the active off-shoot fault. Whether this phenomenon is a general nature regarding strike-slip faults or a special case in the Yamasaki fault will be an important problem to be solved in future. However, we might consider, at least qualitatively, that microearthquakes along clear and active strike-slip faults are closely related to the slip of the fault, and their mechanisms are not incompatible with geological evidences. In other words, it is likely that the orientation of fracture surface of a microearthquake is, to some extent, determined by the existent faults.

Here, it seems interesting to examine the activity and mechanism of much larger earthquakes in this area along the Yamasaki fault system, in comparison with microearthquakes. In 1961, an earthquake swarm occurred at the central portion of the Yamasaki fault, which seems to correspond to $A$ and $B$ regions. Magnitude of the main shock was 6.3. After a detailed investigation ljy Watanabe ${ }^{36)}$, mechanisms of some earthquakes, main shock, 2 foreshocks and one aftershock, show four-quadrant type with vertical null vector. The directions of one nodal plane of these earthquakes were nearly $\mathrm{E} 45^{\circ} \mathrm{S}-\mathrm{W} 45^{\circ} \mathrm{N}$. These results coincide well with those of microearthquakes in A region as mentioned above. Accordingly, it may be considered that earthquake mechanisms are the same along the Yamasaki fault from microearthquakes up to prett! large earthquakes $(M>6)$. Going back to a much older past, a very large earthquake $(M=7.9)$ is estimated to have occurred in this area in $868^{17}$ ). It might be a large scale slipping of this fault. Anyway, it is supposed that the Yramasaki fault is an active fault which has a possibility of generation of large earthquakes.

Mechanisms of dip-slip type are quite few in A to $\mathrm{E}$ regions. Three earthquakes in $\mathrm{C}$ region and an earthquake swarm in $F$ region are exceptional examples. And moreover, it seems to mean that these earthquakes did not occur at the central part of the fault zone, but at the end of the fault where the geological structure is supposedly complicated. The same phenomenon was pointed out by Nishida ${ }^{18)}$ in the case of small earthquakes $(M \geqslant 2.5)$.

Difference in mechanisms of microearthquakes between the Yamasaki fault zone, and $\mathrm{G}$ and $\mathrm{H}$ regions is remarkable. Although tectonic conditions in $\mathrm{G}$ and $\mathrm{H}$ regions are not so clear as in the Yamasaki fault zone, the results in the present analyses seem to suggest that complexity of earthquake mechanism has some tectonic reasons. More precise investigation is necessary in these regions.

A close relation between the earthquake occurrence and geological structure seems to have been strengthened by analysing mechanisms of microearthquakes. So far, it is apt to consider that geological structures are only the phenomena on or mear the earth's surface and they are not directly related to earthquake occurrences which are phenomena at much deeper interior. Above results, however, suggest that both phenomena are closely connected, and that geological and even topographical structures have their roots at much deeper regions.

\section{Conclusions}

1) In order to examine relations between the mechanism of microearthquake and 
geological structure, and then to investigate the tectonic meanings of microearthquake generation, an analysis was made in an area surrounding the Yamasaki fault. Along the Yamasaki fault which is a typical strike-slip fault, almost all earthquakes showed the mechanism of strike-slip type with the maximum pressure axis approximately in horizontal and $E-W$ direction.

2) On the other hand, in a belt-like zone of high microseismicity which makes coniugate direction with the Yamasaki fault and in which geological structure is supposed somewhat complicated, mechanisms of microearthquakes were also complicated, including strike-slip, thrust and even normal fault types.

3) The direction of strike of one nodal plane seems consistently connected to direction of the fault in the corresponding region. This consideration is supported by a fact that, in B region where an active off-shoot of the Yamasaki fault is observed, fault plane solutions showed systematic deviation from regions along the main Yamasaki fault.

4) It may be considered from these results that seismicity and mechanism of microearthquakes are closely related to geological structures. This may mean that activity of microearthquakes is a manifestation at the present time of the tectonic movement which has continued for a long geologic time up to the present.

\section{Acknowledgment}

The authors are indebted to Prof. K. Huzita, Osaka City University, for his valuable advices on geological problems. The authors thank to Prof. H. Miki and staff members of the Abuyama Seismological Observatory for their generous permission to analyse its seismograms.

\section{References}

1) Hashizume, M., K. Oike and Y. Kishimoto: Investigation of Microearthquakes in Kinki District -Seismicity and Mechanism of Their Occurrence-, Bull. Disas. Prev. Res Inst., Kyoto Univ., Vol. 15, Part 3, 1966, 35-47.

2) Kishimoto, Y., M. Hashizume, M. and R. Nishida: Recent Seismicity of Microearthquakes in the Northern Part of Kinki District, Ann. Disas. Prev. Res. inst., Kyoto Univ., No. 11, 1968, 75-89 (in Japanese).

3) Hashizume, M.: Investigation of Microearthquakes-On Seismiciry-, Bull. Disas. Prev. Res. Inst., Kyoto Univ, Vol. 19, Part 2, 1969, 67-85.

4) Huzita, K., Y. Kishimoto and K. Shiono: Neotectonics and Seismicity in the Kinki Area, South. west Japan, J. Geoscience, Osaka City Univ., Vol. 16, 1973, 93-124.

5) Ichikawa, M.: Statistical Investigation of Mechanism of Farthquakes Occurring in and Near Japan and Some Related Problems, Kenkyu-Jiho, JMA, Vol. 18, 1966, 83-154 (in Japanese).

6) Ichikawa, M.: Reanalyses of Mechanism of Earthquakes which Occurred in and near Japan, and Statistical Studies on the Nodal Plane Solutions Oltained, 1926-1968, Geophys. Mag., Vol. 35, 1971. 207-274

7) loc. cit. 1).

8) Hashizume, M.: Investigation of Microearthquakes-On Earthquake Occurrence in the Crust-, Bull. Disas. Prev. Res. Inst., Kyoto Univ., Vol. 20, Part 2, 1970, 65-94. 
9) Okano, K. and I. Hirano: Micro-Earthquakes Occurring in the Vicinity of Kyoto (2), Ann. Disas. Prev. Res. Inst., Kyoto L'niv., No. 9, 1966, 21-26 (in Japanese).

10) Nishida, R.: Earthquake Generating Stress in Eastern Chugoku and Northern Kinki Districts, Southwest Japan, Bull. Disas. Prev. Res. Inst., Kyoto Univ., Vol, 22, Part 3, 1973, 197-233.

11) Huzita, K. et al.: Geology and Geomorphology of the Rokko Area, Kinki District, Japan, with Special Reference to Quaternary Tertonics, J. Creoscience, Osaka City Univ.. Vol. 14, 1971, 71-124.

12) loc, cit, 10).

13) loc. cit. 10).

14) loc. cit. 10).

15) loc. cit. 4).

16) Watanabe, H.: On the Sequence of Earthquakes, Special Contr., Geophys. Inst., Kyoto Univ., No. 4, 1964, 153-192.

17) Tokyo Astronomical Obs.: Rika-Nenpyo, Maruzen co., 1973.

18) Jor. cit. 10). 